University of Wollongong

Research Online

Australian Institute for Innovative Materials -

Papers

Australian Institute for Innovative Materials

$1-1-2013$

Flux pinning mechanism in BaFe1.9Ni0.1As2 single crystals: Evidence for fluctuation in mean free path induced pinning

M Shahbazi-Manshadi

University of Wollongong, msm979@uowmail.edu.au

X L Wang

University of Wollongong, xiaolin@uow.edu.au

K Y Choi

Sogang University ,Tohoku University,Seoul National University

SX. Dou

University of Wollongong, shi@uow.edu.au

Follow this and additional works at: https://ro.uow.edu.au/aiimpapers

Part of the Engineering Commons, and the Physical Sciences and Mathematics Commons

Research Online is the open access institutional repository for the University of Wollongong. For further information contact the UOW Library: research-pubs@uow.edu.au 


\title{
Flux pinning mechanism in BaFe1.9Ni0.1As2 single crystals: Evidence for fluctuation in mean free path induced pinning
}

\author{
Abstract \\ The flux pinning mechanism of BaFe1.9Ni0.1As2 superconducting crystals have been investigated \\ systematically by magnetic measurements up to $13 \mathrm{~T}$ at various temperatures. The field dependence of \\ the critical current density, Jc, was analysed within the collective pinning model. A remarkably good \\ agreement between the experimental results and theoretical dl pinning curve is obtained, which indicates \\ that pinning in $\mathrm{BaFe} 1.9 \mathrm{Ni0} .1 \mathrm{As} 2$ crystal originates from spatial variation of the mean free path. Moreover, \\ the normalized pinning force density, Fp, curves versus h1/4B/Birr (Birr is the irreversibility field) were \\ scaled using the Dew-Hughes model. Analysis suggests that point pinning alone cannot explain the \\ observed field variation of Fp.

\section{Keywords} \\ pinning, mechanism, bafe1, 9ni0, 1as2, flux, single, mean, crystals, evidence, fluctuation, induced, path, \\ free

\section{Disciplines} \\ Engineering | Physical Sciences and Mathematics

\section{Publication Details} \\ Shahbazi-Manshadi, M., Wang, X., Choi, K. \& Dou, S. X. (2013). Flux pinning mechanism in \\ BaFe1.9Ni0.1As2 single crystals: Evidence for fluctuation in mean free path induced pinning. Applied \\ Physics Letters, 103 (3), 032605-1-032605-4.
}




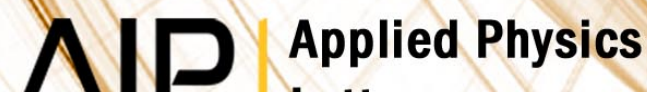 \\ Letters}

Flux pinning mechanism in BaFe1.9Ni0.1As2 single crystals: Evidence for fluctuation in mean free path induced pinning

M. Shahbazi, X. L. Wang, K. Y. Choi, and S. X. Dou

Citation: Appl. Phys. Lett. 103, 032605 (2013); doi: 10.1063/1.4813113

View online: http://dx.doi.org/10.1063/1.4813113

View Table of Contents: http://apl.aip.org/resource/1/APPLAB/v103/i3

Published by the AIP Publishing LLC.

Additional information on Appl. Phys. Lett.

Journal Homepage: http://apl.aip.org/

Journal Information: http://apl.aip.org/about/about_the_journal

Top downloads: http://apl.aip.org/features/most_downloaded

Information for Authors: http://apl.aip.org/authors

\section{ADVERTISEMENT}
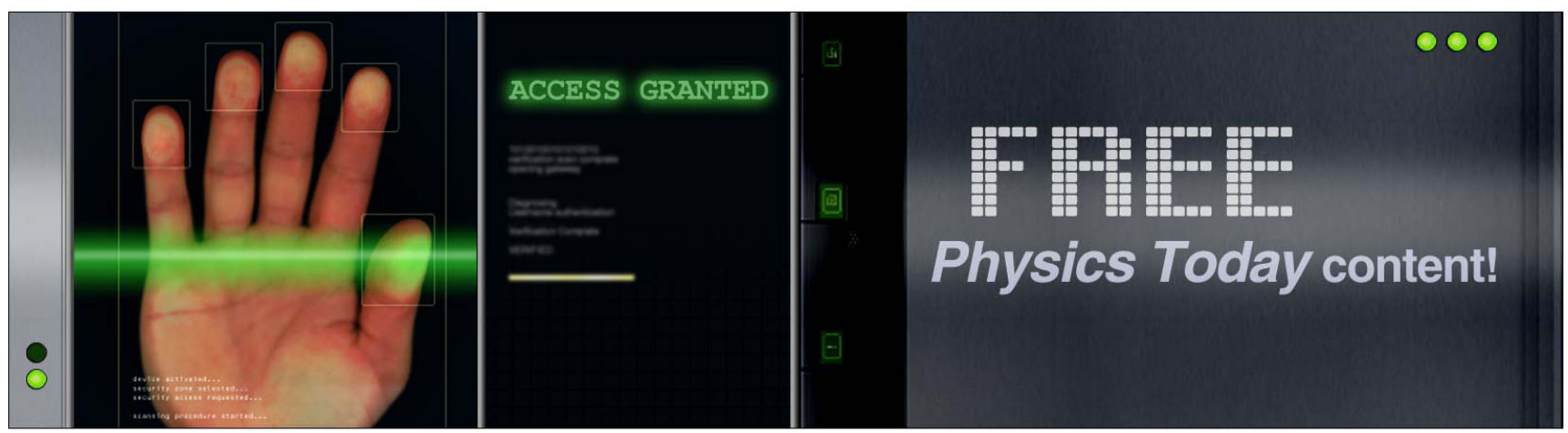


\title{
Flux pinning mechanism in $\mathrm{BaFe}_{1.9} \mathrm{Ni}_{0.1} \mathrm{As}_{2}$ single crystals: Evidence for fluctuation in mean free path induced pinning
}

\author{
M. Shahbazi, ${ }^{1}$ X. L. Wang, ${ }^{1, a)}$ K. Y. Choi, ${ }^{2}$ and S. X. Dou ${ }^{1}$ \\ ${ }^{1}$ Institute for Superconducting and Electronic Materials, University of Wollongong, North Wollongong, \\ NSW 2519, Australia \\ ${ }^{2}$ Center for Novel States of Complex Materials Research, Department of Physics and Astronomy, \\ Seoul National University, Seoul 151-747, South Korea
}

(Received 10 April 2013; accepted 17 June 2013; published online 19 July 2013)

\begin{abstract}
The flux pinning mechanism of $\mathrm{BaFe}_{1.9} \mathrm{Ni}_{0.1} \mathrm{As}_{2}$ superconducting crystals have been investigated systematically by magnetic measurements up to $13 \mathrm{~T}$ at various temperatures. The field dependence of the critical current density, $J_{\mathrm{c}}$, was analysed within the collective pinning model. A remarkably good agreement between the experimental results and theoretical $\delta l$ pinning curve is obtained, which indicates that pinning in $\mathrm{BaFe}_{1.9} \mathrm{Ni}_{0.1} \mathrm{As}_{2}$ crystal originates from spatial variation of the mean free path. Moreover, the normalized pinning force density, $F_{p}$, curves versus $h=B / B_{\text {irr }}\left(B_{\text {irr }}\right.$ is the irreversibility field) were scaled using the Dew-Hughes model. Analysis suggests that point pinning alone cannot explain the observed field variation of $F_{p}$. C 2013 AIP Publishing LLC. [http://dx.doi.org/10.1063/1.4813113]
\end{abstract}

Study of the vortex mechanisms in pnictides is crucial for practical applications due to relatively high critical temperature, $T_{c}$, high upper critical field, ${ }^{1} B_{\mathrm{c} 2}$, high $J_{c}$, very high intrinsic pinning potential, ${ }^{2}$ and nearly isotropic superconductivity $^{3,4}$ of these compounds. The 122 family, $A \mathrm{Fe}_{2} \mathrm{As}_{2}$ has attracted great interest for the study of superconducting properties due to their simple crystal structures and possibility of growing large single crystals. ${ }^{5,6}$

For some superconductors, the $J_{\mathrm{c}}$ obtained from magnetic hysteresis loops (MHLs), increases with magnetic field after the first peak of penetration field. This is the so-called second magnetization peak (SMP) or fishtail effect. In conventional low temperature superconductors, e.g., $\mathrm{MgB}_{2},{ }^{7} \mathrm{Nb}_{3} \mathrm{Sn},{ }^{8}$ etc., SMP corresponds to a hump feature in $J_{\mathrm{c}}(B)$ far below the $B_{\mathrm{c} 2}$ while peak effect (PE) is realized to happen near $B_{\mathrm{c} 2} \cdot{ }^{9}$ It is suggested that the PE is associated with the rapid softening of the flux line lattice. ${ }^{10}$ In high temperature superconductors, different mechanisms including inhomogeneity of the sample, ${ }^{11}$ Dynamic effects, ${ }^{12}$ structural phase transition in the vortex lattice, ${ }^{13}$ vortex order-disorder phase transition, ${ }^{14,15}$ and cross over from elastic to plastic creep $^{16}$ have been proposed to explain the SMP. It is reported SMP occur in $\mathrm{SmFeAsO}_{0.9} \mathrm{~F}_{0.1}$ (Ref. 17) as a result of weak and collective pinning of the system. The SMP observed only for the samples near optimally doping for $\mathrm{NdFeAsO}_{0.85},{ }^{18} \mathrm{Ba}\left(\mathrm{Fe}_{1-x} \mathrm{Co}_{\mathrm{x}}\right)_{2} \mathrm{As}_{2}$ (Refs. 19-22) and weak and collective pinning are concluded for most studies. The SMP has also been observed in optimally doped $\mathrm{Ba}_{1-x} \mathrm{~K}_{x} \mathrm{Fe}_{2} \mathrm{As}_{2}{ }^{19,23}$ However, it is worth to note that most form of in-homogeneity like $T_{\mathrm{c}}$ variation, impurity phase, doping variation, etc., might prevent the occurrence of SMP. ${ }^{24}$ For example, an under-doped $\mathrm{Ba}_{1-x} \mathrm{~K}_{x} \mathrm{Fe}_{2} \mathrm{As}_{2}$ system does not show the SMP. Also SMP has been reported for $\mathrm{Ba}\left(\mathrm{Fe}_{1-x} \mathrm{Ni}_{x}\right)_{2} \mathrm{As}_{2},{ }^{19,25} \mathrm{LiFeAs}^{26}{ }^{26} \mathrm{FeTe}_{1-x} \mathrm{Se}_{x}$ (Ref. 27), and $\mathrm{PrFeAsO}_{0.6} \mathrm{~F}_{0.1} \cdot{ }^{28}$ The observed SMP for electron and hole doped Ba-122 crystals for $H / / \mathrm{c}^{19}$ was disappeared for $H / / \mathrm{ab}$,

\footnotetext{
${ }^{\text {a) }}$ Author to whom correspondence should be addressed. Electronic mail: xiaolin@uow.edu.au
}

indicating an anisotropic effect of flux pinning for these compounds. ${ }^{19}$

There are two main pinning mechanisms in type II superconductors: (I) $\delta l$ pinning from spatial variation in the charge carrier mean free path, $l$, and (II) $\delta T_{c}$ pinning due to randomly distributed spatial variation in $T_{c}$. It has been reported that strong pinning centres in $\mathrm{PrFeAsO}_{0.9}$ and $\mathrm{NdFeAsO} \mathrm{O}_{0.9} \mathrm{~F}_{0.1}$ arise from oxygen deficiency and dopant atoms, which results in pinning by local variations in the mean-free path. ${ }^{29}$ Strong intrinsic pinning due to structural domains in the superconducting orthorhombic phase ${ }^{30}$ of $\mathrm{Ba}\left(\mathrm{Fe}_{1-x} \mathrm{Co}_{x}\right)_{2} \mathrm{As}_{2}$ is also observed. Similar results were found for $\mathrm{BaFe}_{1.8} \mathrm{Co}_{0.2} \mathrm{As}_{2}$, where the temperature and field dependence of $J_{c}$ were attributed to the inhomogeneous distribution of Co atoms. ${ }^{31}$ Furthermore, It has been suggested that the very large $J_{c}$ and fishtail effect at high temperature below $T_{c}$ in $\mathrm{Ba}_{0.6} \mathrm{~K}_{0.4} \mathrm{Fe}_{2} \mathrm{As}_{2}$ had originated from the smallsize normal core pinning centres. ${ }^{23}$

In this work, we present a systematic study of the flux pinning mechanism of $\mathrm{BaFe}_{1.9} \mathrm{Ni}_{0.1} \mathrm{As}_{2}$ crystals.

Single crystal of $\mathrm{BaFe}_{1.9} \mathrm{Ni}_{0.1} \mathrm{As}_{2}$ was prepared by a selfflux method. ${ }^{32,33}$ For magnetic measurements, the as-grown single crystal was cleaved and cut into a rectangular shape. The present $\mathrm{BaFe}_{1.9} \mathrm{Ni}_{0.1} \mathrm{As}_{2}$ sample has dimensions of $1.56 \times 2.82 \times 0.06 \mathrm{~mm}^{3}$. Magnetization loops were collected in different magnetic fields with $B / / \mathrm{c}$ and at temperatures down to $3 \mathrm{~K}$ using a superconducting quantum interference device vibrating sample magnetometer (SQUID-VSM, Quantum Design).

The inset of Fig. 1 shows the temperature dependence of the magnetization measured after zero-field-cooling (ZFC) and field-cooling (FC) of the $\mathrm{BaFe}_{1.9} \mathrm{Ni}_{0.1} \mathrm{As}_{2}$ crystal, with a field of $200 \mathrm{Oe}$ for $\mathrm{B} / \mathrm{/c}$. The $T_{\mathrm{c}}$ of $17.7 \mathrm{~K}$ was determined from the onset of the transition. The MHLs of the $\mathrm{BaFe}_{1.9} \mathrm{Ni}_{0.1} \mathrm{As}_{2}$ crystal at $3 \mathrm{~K}<T<15 \mathrm{~K}$ for $B / / c$, are shown in the main panel of Fig. 1. The almost perfect symmetry of the MHLs with respect to the $\mathrm{x}$-axis indicates that bulk pinning is dominant. ${ }^{25}$ The minimum in the magnetization, which is located slightly above 


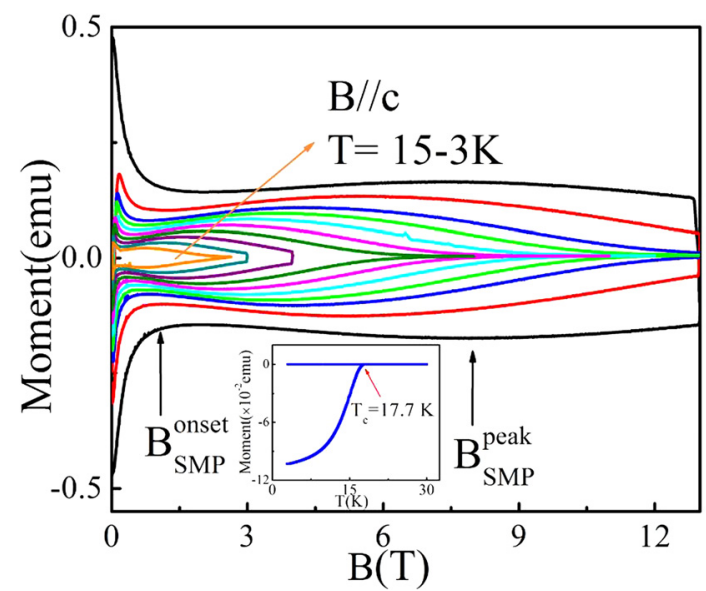

FIG. 1. MHLs at various temperatures for $B / / c$. Inset: Temperature dependence of the magnetic susceptibility.

zero field in a given MHLs, characterizes the onset of SMP. At this field, the applied magnetic field penetrates completely into the bulk sample after ZFC. ${ }^{34}$ The SMP can be observed at all temperatures below $15 \mathrm{~K}$, similar to the behaviour of $(\mathrm{Ba}, \mathrm{K}) \mathrm{Fe}_{2} \mathrm{As}_{2}$ (Ref. 23) and $\mathrm{BaFe}_{1-x} \mathrm{Co}_{x} \mathrm{As}_{2}$ crystals. ${ }^{31}$ Arrows indicate the onset $\left(B_{S M P}^{\text {onset }}\right)$ and peak $\left(B_{S M P}^{\text {peak }}\right)$ positions of the SMP in Fig. 1 for $\mathrm{T}=3 \mathrm{~K}$. In some of the conventional superconductors, such as $\mathrm{MgB}_{2}$ (Ref. 7) and $\mathrm{Nb}_{3} \mathrm{Sn},{ }^{8}$ the $\mathrm{PE}$ occurs at a field close to the $B_{\mathrm{c} 2}$. It is believed that the PE is associated with the metastability of an underlying first-order vortex melting transition, where softening of the vortex due to the thermal fluctuation leads to a better accommodation of the pinning centres by the vortex lattice. ${ }^{8}$ This explanation of the $\mathrm{PE}$ in $\mathrm{Nb}_{3} \mathrm{Sn}$ does not appear to be applicable to the PE observed in cuprates and pnictides, in which PE occurs far from the normal phase boundary. In the case of cuprates, it has been suggested that a first-order disorder driven transition is responsible for SMP. ${ }^{35}$ Salem-Sugui, Jr. et al. ${ }^{36}$ studied the vortex dynamics of an over-doped $\mathrm{BaFe}_{1.82} \mathrm{Ni}_{0.18} \mathrm{As}_{2}$ crystal by measuring flux creep over the SMP and suggested that the SMP could not arise due to the softening in the vortex pinning prior to melting nor from a change in the pinning regime within a collective model. Also, their study of an optimally doped $\mathrm{BaFe}_{1.9} \mathrm{Ni}_{0.1} \mathrm{As}_{2}$ crystal did not show any evidence of a pinning crossover occurring near the SMP of the MHLs. ${ }^{25}$ Magnetic studies by Prozorov et al. ${ }^{21}$ and Shen et al. ${ }^{20}$ interpreted the SMP as signifying a crossover from elastic to plastic vortex creep. The same result was obtained by Kopeliansky et al., who suggested that the SMP is associated with a vortex structural phase transition from a rhombic to a square lattice. ${ }^{22}$

The, $J_{c}$, values were extracted from the MHLs, using Bean's model, ${ }^{37}$ where $J_{\mathrm{c}}(B)=20 \times \Delta M(B) /(l(1-l / 3 w))$, with $l$ and $w$ being the sample dimensions perpendicular to the applied magnetic field, $l<w$, and $\Delta M$ is the width of the hysteresis loops.

Figure 2 shows the field dependence of $J_{\mathrm{c}}$ at different temperatures for $B / / \mathrm{c}$. The obtained value of $J_{\mathrm{c}}=0.14$ $\times 10^{6} \mathrm{~A} / \mathrm{cm}^{2}$ at zero field and $\mathrm{T}=10 \mathrm{~K}$ is comparable with the reported value of $J_{\mathrm{c}}=0.23 \times 10^{6}$ for the optimally doped sample. ${ }^{19}$ The inset of Figure 2 illustrates a normalized $J_{\mathrm{c}}$ versus $B$ plot at selected temperatures. The onset position, $B_{S M P}^{\text {onset }}$, and peak position, $B_{S M P}^{\text {peak }}$, of the SMP are marked for

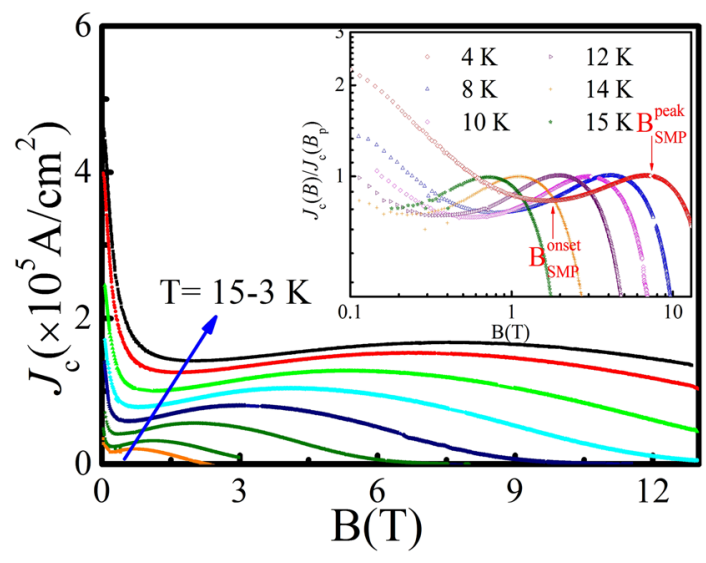

FIG. 2. Field dependence of $J_{\mathrm{c}}$ at different temperatures for $B / / \mathrm{c}$. Inset: Field dependence of the normalized $J_{\mathrm{c}}$ at different temperatures for $B / / c$.

$T=4 \mathrm{~K}$. The position of SMP shifts toward lower field with increasing temperature, for example, $B_{S M P}^{\text {peak }}$ decreases considerably from $6.8 \mathrm{~T}$ at $4 \mathrm{~K}$ to $0.7 \mathrm{~T}$ at $15 \mathrm{~K}$, but $B_{S M P}^{\text {onset }}$ drops slowly from $1.7 \mathrm{~T}$ at $4 \mathrm{~K}$ to $0.2 \mathrm{~T}$ at $15 \mathrm{~K}$, respectively. Similar behaviour was observed for $R E \mathrm{Ba}_{2} \mathrm{Cu}_{3} \mathrm{O}_{7-\delta} .{ }^{38} \mathrm{It}$ is likely that the SMP has the same mechanism in both compounds. $^{23}$

In Fig. 3, we present the vortex phase diagram of the $\mathrm{BaFe}_{1.9} \mathrm{Ni}_{0.1} \mathrm{As}_{2}$ crystal. Three characteristic fields, $B_{\text {irr }}$, $B_{S M P}^{\text {peak }}$, and $B_{S M P}^{\text {onset }}$, were determined from magnetic measurements as shown by the solid symbols in Fig. 3. It is clear that the $B_{\text {irr }}-T, B_{S M P}^{\text {peak }}-T$, and $B_{S M P}^{\text {onset }}-T$ are temperature dependent. The large area between $B_{\text {irr }}-T$ and $B_{S M P}^{\text {peak }}-T$ suggests that the vortex dissipation is through plastic motion in this area, as proposed by Shen et al. for optimally Co doped $\mathrm{BaFe}_{2} \mathrm{As}_{2} .{ }^{20}$ The dashed lines represent the fitting curves using $B(T)=A\left(1-T / T_{\mathrm{c}}\right)^{n}$, with $n$ being a fitting parameter. All the curves were well fitted using the expression with $n=1.9$ for $B_{S M P}^{\text {onset }}$ and $n=1.4$ for $B_{S M P}^{\text {peak }}$ and $B_{\text {irr }}$. These values are similar to the values obtained for $\mathrm{BaFe}_{2-x} \mathrm{Co}_{x} \mathrm{As}_{2}$ crystal. $^{20}$

In order to assess the nature of the pinning mechanisms in more detail, it is useful to look at the variation of the vortex pinning force, $F_{p}=B \times J_{c}$, with the magnetic field. In Fig. 4 , we plot the normalized pinning force, $F_{p}^{\text {norm }}$ $=F_{p} / F_{p}^{\max }$, as a function of the reduced field, $h=B / B_{\text {irr }}$.

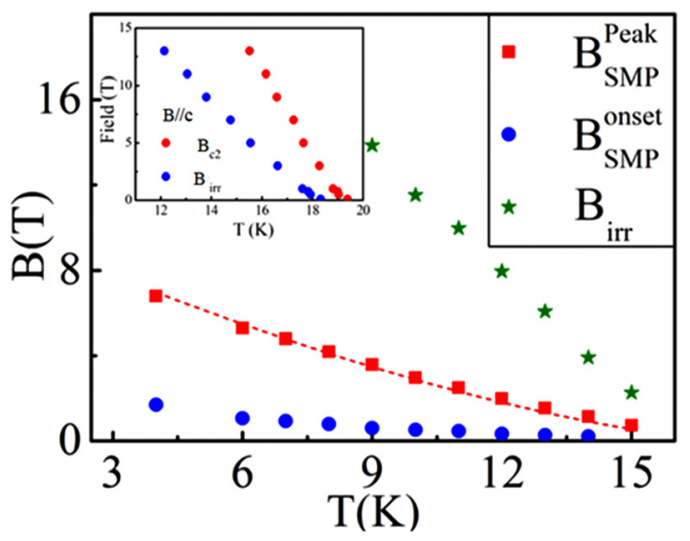

FIG. 3. Vortex phase diagram of $\mathrm{BaFe}_{1.9} \mathrm{Ni}_{0.1} \mathrm{As}_{2}$ single crystal determined from magnetic measurements for $\mathrm{B} / \mathrm{c}$. Inset: Temperature dependence of $\mathrm{B}_{\mathrm{c} 2}$ and $\mathrm{B}_{\mathrm{irr}}$ obtained from $\rho-T$ curves for $\mathrm{B} / / \mathrm{c}$. 


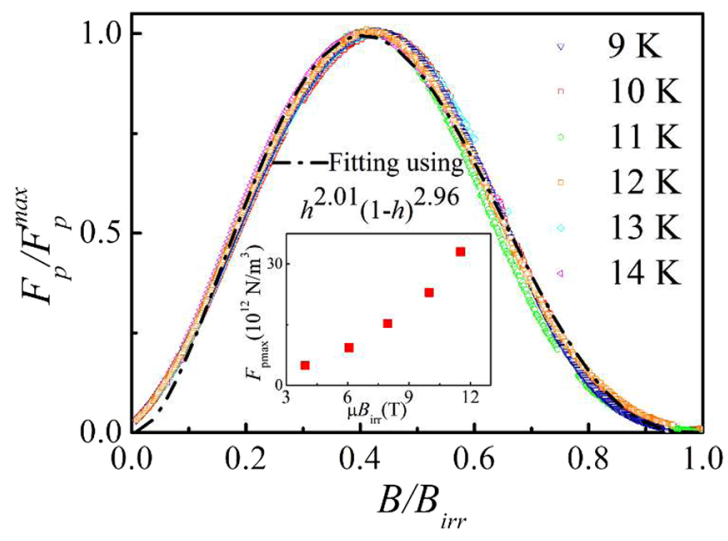

FIG. 4. Normalized flux pinning force $F_{p}^{n o r m}=F_{p} / F_{p}^{\max }$ as a function of reduced field, $h=B / B_{\text {irr }}$. The dashed-dotted line represents the fitting curve $h^{2.01}(1-h)^{2.96}$. Inset: Field dependence of the maximum pinning force.

The $B_{\text {irr }}$ is estimated using the criterion of $J_{\mathrm{c}}<100 \mathrm{~A} / \mathrm{cm}^{2}$. It should be noted the scaling of the normalized pinning force is done based on the reduced field by $B_{\text {irr }}$ with $h=B / B_{\text {irr }}$ instead of $B_{\mathrm{c} 2}$ with $h=B / B_{\mathrm{c} 2}$ due to the facts that the difference between $B_{\mathrm{c} 2}$ and $B_{\text {irr }}$ is sizable, and is more significant at low temperature regime in the case of pnictides, $\mathrm{MgB}_{2}$ and cuprates. $^{7,29,32,38,39}$ The temperature dependence of $B_{\mathrm{c} 2}$ and $B_{\text {irr }}$ obtained from $\rho$-T curves (see the inset in Fig. 3) clearly reveal the $B_{\text {irr }}$ is far below the $B_{\mathrm{c} 2}$. Note that all the $F_{p}^{\text {norm }}$ curves for $9 \mathrm{~K} \leq T \leq 14 \mathrm{~K}$ collapse into one unified curve. We fit these data using the Dew-Hughes formula, $F_{p} \propto h^{p}$ $(1-h)^{q},{ }^{40}$ where $p$ and $q$ are two parameters whose values depend on the origin of the pinning mechanism. The Dew-Hughes fit is shown by the black dashed-dotted line in Fig. 4 with $p=2.01$ and $q=2.96$. The value $p /(p+q) \approx 0.4$ matches well with the peak positions in the $F_{p}^{\text {norm }}$ versus $h$ plots. According to the Dew-Hughes model, in the case of $\delta l$ pinning for a system dominated just by point pinning, $p=1$ and $q=2$, with $F_{p}^{\text {norm }}$ occurring at $h_{\max }=0.33$. Pinning due to grain boundaries leads to $h_{\max } \approx 0.2$, while in a system in which variation in the superconducting order parameter controls the pinning mechanism, $h_{\max } \approx 0.7 .^{40,41}$ In the case of $\delta T_{c}$ pinning, the maximum of $F_{p}$ is expected to be located at higher $h$ values. For example, for point pinning, the maximum is expected at $h=0.67$ with $p=2$ and $q=1$. For surface pins, the maximum exists at $h=0.6, p=1.5$, and $q=1$, and for volume pins, $h=0.5$ with $p=1$ and $q=1$. In our case, $h_{\max }=0.4$, indicating that point pinning alone cannot explain the pinning mechanism in the $\mathrm{BaFe}_{1.9} \mathrm{Ni}_{0.1} \mathrm{As}_{2}$ crystal. Similar analysis has been done for $\mathrm{Ba}_{0.68} \mathrm{~K}_{0.32} \mathrm{Fe}_{2} \mathrm{As}_{2}$ $\left(h_{\max }=0.43\right), \mathrm{BaFe}_{1.85} \mathrm{Co}_{0.15} \mathrm{As}_{2}\left(h_{\max }=0.37\right)$, and $\mathrm{BaFe}_{1.91}$ $\mathrm{Ni}_{0.09} \mathrm{As}_{2}\left(h_{\max }=0.33\right)$ by Sun et al. ${ }^{19}$ They noticed that $B_{c 2}$ and $B_{S M P}^{\text {peak }}$ decrease faster with decreasing temperature for $\mathrm{BaFe}_{1.91} \mathrm{Ni}_{0.09} \mathrm{As}_{2}$ compared to $\mathrm{Ba}_{0.68} \mathrm{~K}_{0.32} \mathrm{Fe}_{2} \mathrm{As}_{2}$ and $\mathrm{BaFe}_{1.85} \mathrm{Co}_{0.15} \mathrm{As}_{2}$ crystals, which is related to the inhomogeneity in $\mathrm{BaFe}_{1.91} \mathrm{Ni}_{0.09} \mathrm{As}_{2}$. The fact that the strongest pinning is in $\mathrm{Ba}_{0.68} \mathrm{~K}_{0.32} \mathrm{Fe}_{2} \mathrm{As}_{2}$ among these three systems indicates that inhomogeneous distribution of dopants or As deficiency cannot play a crucial role in determining strong pinning in pnictides. Yang et al. proposed that the obtained value of $h_{\max }=0.33$ should be attributed to the small-size normal cores, as in the case of arsenic deficiency in $(\mathrm{Ba}, \mathrm{K}) \mathrm{Fe}_{2} \mathrm{As}_{2}$ crystal. ${ }^{23}$ In the case of $\mathrm{BaFe}_{1.8} \mathrm{Co}_{0.2} \mathrm{As}_{2}$, a peak at

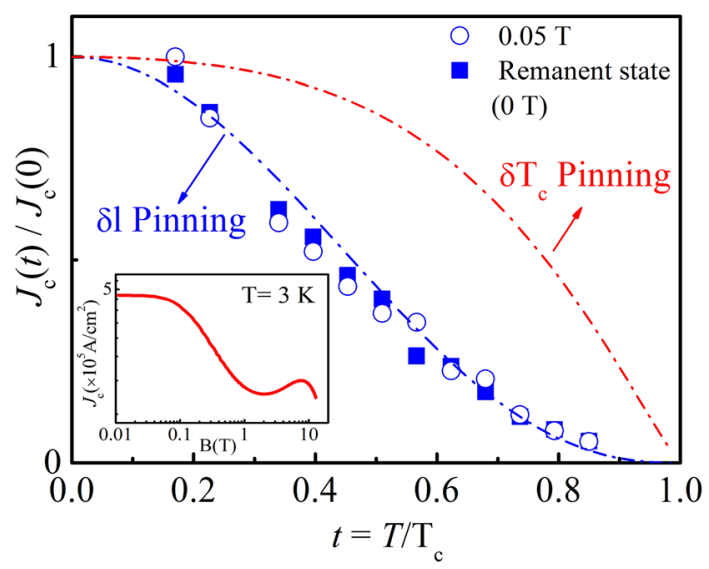

FIG. 5. Temperature dependence of the normalized measured $J_{\mathrm{c}}$ at $0.05 \mathrm{~T}$ (open circle) and $0 \mathrm{~T}$ (solid square). Inset: Field dependence of $J_{\mathrm{c}}$ at $\mathrm{T}=3 \mathrm{~K}$.

$h_{\max } \approx 0.45$ was suggested to be related to the inhomogeneous distribution of Co ions. ${ }^{31}$ In particular, the fact that, in our case, the maximum in $F_{p}$ occurs at $h<0.5$ indicates that the pinning centres in $\mathrm{BaFe}_{1.9} \mathrm{Ni}_{0.1} \mathrm{As}_{2}$ are of the $\delta l$ type, while for $\delta T_{\mathrm{c}}$ pinning, it is expected that the maximum would occur at $h>0.5$.

With the aim of understanding more about the origins of the pinning in $\mathrm{BaFe}_{1.9} \mathrm{Ni}_{0.1} \mathrm{As}_{2}$ crystal, the experimental results were analysed using collective pinning theory. According to the theoretical approach proposed by Griessen et al. ${ }^{42}$ in the case of $\delta l$ pinning, $J_{\mathrm{c}}(t) / J_{\mathrm{c}}(0) \propto\left(1-t^{2}\right)^{5 / 2}\left(1+t^{2}\right)^{-1 / 2}$, while for $\delta T_{\mathrm{c}}$ pinning, $J_{\mathrm{c}}(t) / J_{\mathrm{c}}(0) \propto\left(1-t^{2}\right)^{7 / 6}\left(1+t^{2}\right)^{5 / 6}$, where $t=T / T_{\mathrm{c}}$. It should be noted that the flux pinning is two dimensional in such thin film, as the correlation length along the flux lines exceeds the film thickness. ${ }^{43}$ Figure 5 shows a comparison between the experimental $J_{\mathrm{c}}$ values and the theoretically expected variation within the $\delta l$ and $\delta T_{\mathrm{c}}$ pinning mechanisms at $0.05 \mathrm{~T}$ (open circle) and $0 \mathrm{~T}$ (the so called remanent state shown by solid square). The $J_{\mathrm{c}}(t)$ values have been obtained from the $J_{\mathrm{c}}(B)$ curves at several temperatures. A remarkably good agreement between the experimental results and theoretical $\delta l$ pinning curve is obtained. It is likely that pinning in $\mathrm{BaFe}_{1.9} \mathrm{Ni}_{0.1} \mathrm{As}_{2}$ crystal originates from spatial variation of the mean free path. Our observation of the dominant $\delta l$ pinning is in good agreement with the reported $\delta 1$ pinning mechanism for $\mathrm{K}_{x} \mathrm{Fe}_{2-y} \mathrm{Se}_{2}, \mathrm{FeSe}_{0.5} \mathrm{Te}_{0.5}$ and $\mathrm{FeTe}_{0.7} \mathrm{Se}_{0.3}$ crystals at low magnetic field.

In summary, we have observed the SMP effect in $\mathrm{BaFe}_{1.9} \mathrm{Ni}_{0.1} \mathrm{As}_{2}$ crystal. The onset and peak positions of the SMP move to lower magnetic field as the temperature is raised. Analysis using the Dew-Hughes model suggests that point pins alone cannot explain the observed field variation of the pinning force density. The maximum in $F_{p}$ at $h<0.5$ indicates that the pinning centres in $\mathrm{BaFe}_{1.9} \mathrm{Ni}_{0.1} \mathrm{As}_{2}$ are of the $\delta l$ type, while for $\delta T_{\mathrm{c}}$ pinning, it is expected that the maximum would occur at $h>0.5$. In addition, a good agreement between experimental and theoretical fitting using $\delta l$ pinning is obtained based on collective flux pinning model.

This work was supported by the Australian Research Council through Discovery Projects, DP1094073 and DP0770205. M. Shahbazi would like to thank Professor Jin Zou at University of Queensland for his support. K.Y. Choi 
was supported by the Basic Science Research Program (Grant No. 2012-008233) and Creative Research Initiative (Grant No. 2010-0018300) funded by the Korean Federation of Science and Technology Societies.

${ }^{1}$ F. Hunte, J. Jaroszynski, A. Gurevich, D. C. Larbalestier, R. Jin, A. S. Sefat, M. A. McGuire, B. C. Sales, D. K. Christen, and D. Mandrus, Nature 453, 903-905 (2008).

${ }^{2}$ X.-L. Wang, S. R. Ghorbani, S.-I. Lee, S. X. Dou, C. T. Lin, T. H. Johansen, K. H. Müller, Z. X. Cheng, G. Peleckis, M. Shabazi et al., Phys. Rev. B 82, 024525 (2010).

${ }^{3}$ H. Q. Yuan, J. Singleton, F. F. Balakirev, S. A. Baily, G. F. Chen, J. L. Luo, and N. L. Wang, Nature 457, 565-568 (2009).

${ }^{4}$ M. Shahbazi, X. L. Wang, S. R. Ghorbani, S. X. Dou, and K. Y. Choi, Appl. Phys. Lett. 100, 102601 (2012).

${ }^{5}$ C. Yanchao, L. Xingye, W. Meng, L. Huiqian, and L. Shiliang, Supercond. Sci. Technol. 24, 065004 (2011).

${ }^{6}$ N. Ni, S. L. Bud'ko, A. Kreyssig, S. Nandi, G. E. Rustan, A. I. Goldman, S. Gupta, J. D. Corbett, A. Kracher, and P. C. Canfield, Phys. Rev. B 78, 014507 (2008).

${ }^{7}$ M. Pissas, S. Lee, A. Yamamoto, and S. Tajima, Phys. Rev. Lett. 89, 097002 (2002).

${ }^{8}$ R. Lortz, N. Musolino, Y. Wang, A. Junod, and N. Toyota, Phys. Rev. B 75, 094503 (2007).

${ }^{9}$ S. Sarkar, D. Pal, P. Paulose, S. Ramakrishnan, A. Grover, C. Tomy, D. Dasgupta, B. Sarma, G. Balakrishnan, and D. Paul, Phys. Rev. B 64, 144510 (2001).

${ }^{10}$ S. Bhattacharya and M. Higgins, Phys. Rev. B 49, 10005-10008 (1994).

${ }^{11}$ M. Daeumling, J. M. Seuntjens, and D. Larbalestier, Nature 346, 332 (1990).

${ }^{12}$ L. Krusin-Elbaum, L. Civale, V. Vinokur, and F. Holtzberg, Phys. Rev. Lett. 69, 2280-2283 (1992).

${ }^{13}$ R. Baruch and K. Anton, Phys. Rev. Lett. 83, 844 (1999).

${ }^{14}$ D. Giller, A. Shaulov, R. Prozorov, Y. Abulafia, Y. Wolfus, L. Burlachkov, and Y. Yeshurun, Phys. Rev. Lett. 79, 2542 (1997).

${ }^{15}$ B. Khaykovich, E. Zeldov, D. Majer, T. W. Li, P. H. Kes, and M. Konczykowski, Phys. Rev. Lett. 76, 2555 (1996).

${ }^{16}$ Y. Abulafia, A. Shaulov, Y. Wolfus, R. Prozorov, L. Burlachkov, and V. Yeshurun, Phys. Rev. Lett. 77, 1596 (1996).

${ }^{17}$ H. Yang, C. Ren, L. Shan, and H.-H. Wen, Phys. Rev. B 78, 092504 (2008).

${ }^{18}$ J. D. Moore, L. F. Cohen, Y. Yeshurun, A. D. Caplin, K. Morrison, K. A. Yates, C. M. McGilvery, J. M. Perkins, D. W. McComb, C. Trautmann et al., Supercond. Sci. Technol. 22, 125023 (2009).

${ }^{19}$ D. L. Sun, Y. Liu, and C. T. Lin, Phys. Rev. B 80, 144515 (2009).

${ }^{20}$ B. Shen, P. Cheng, Z. Wang, L. Fang, C. Ren, L. Shan, and H.-H. Wen, Phys. Rev. B 81, 014503 (2010).
${ }^{21}$ R. Prozorov, N. Ni, M. A. Tanatar, V. G. Kogan, R. T. Gordon, C. Martin, E. C. Blomberg, P. Prommapan, J. Q. Yan, S. L. Bud'ko et al., Phys. Rev. B 78, 224506 (2008).

${ }^{22}$ R. Kopeliansky, A. Shaulov, B. Y. Shapiro, Y. Yeshurun, B. Rosenstein, J. J. Tu, L. J. Li, G. H. Cao, and Z. A. Xu, Phys. Rev. B 81, 092504 (2010).

${ }^{23}$ H. Yang, H. Luo, Z. Wang, and H.-H. Wen, Appl. Phys. Lett. 93, 142506 (2008).

${ }^{24}$ J. T. Park, D. S. Inosov, C. Niedermayer, G. L. Sun, D. Haug, N. B. Christensen, R. Dinnebier, A. V. Boris, A. J. Drew, L. Schulz et al., Phys. Rev. Lett. 102, 117006 (2009).

${ }^{25}$ S. Salem-Sugui, Jr., L. Ghivelder, A. D. Alvarenga, L. F. Cohen, H. Luo, and X. Lu, e-print arXiv:1209.1594v1.

${ }^{26}$ A. K. Pramanik, L. Harnagea, C. Nacke, A. U. B. Wolter, S. Wurmehl, V. Kataev, and B. Büchner, Phys. Rev. B 83, 094502 (2011).

${ }^{27}$ M. Bonura, E. Giannini, R. Viennois, and C. Senatore, Phys. Rev. B 85, 134532 (2012).

${ }^{28}$ D. Bhoi, P. Mandal, P. Choudhury, S. Dash, and A. Banerjee, Physica C 471, 258-264 (2011).

${ }^{29}$ C. J. van der Beek, G. Rizza, M. Konczykowski, P. Fertey, I. Monnet, T. Klein, R. Okazaki, M. Ishikado, H. Kito, A. Iyo et al., Phys. Rev. B 81, 174517 (2010).

${ }^{30}$ R. Prozorov, M. A. Tanatar, N. Ni, A. Kreyssig, S. Nandi, S. L. Bud'ko, A. I. Goldman, and P. C. Canfield, Phys. Rev. B 80, 174517 (2009).

${ }^{31}$ A. Yamamoto, J. Jaroszynski, C. Tarantini, L. Balicas, J. Jiang, A. Gurevich, D. C. Larbalestier, R. Jin, S. Sefat, M. A. McGuire et al., Appl. Phys. Lett. 94, 062511 (2009).

${ }^{32}$ Y. Liu, R. K. Kremer, and C. T. Lin, EPL 92, 57004 (2010).

${ }^{33}$ Y. Liu and C. T. Lin, J. Supercond. Novel Magn. 24, 183-187 (2011).

${ }^{34}$ P. Das, A. D. Thakur, A. K. Yadav, C. V. Tomy, M. R. Lees, G. Balakrishnan, S. Ramakrishnan, and A. K. Grover, Phys. Rev. B 84, 214526 (2011).

${ }^{35}$ N. Avraham, B. Khaykovich, Y. Myasoedov, M. Rappaport, H. Shtrikman, D. E. Feldman, T. Tamegai, P. H. Kes, M. Li, M. Konczykowski et al., Nature 411, 451-454 (2001).

${ }^{36}$ S. Salem-Sugui, Jr., L. Ghivelder, A. D. Alvarenga, L. F. Cohen, H. Luo, and X. Lu, Phys. Rev. B 84, 052510 (2011).

${ }^{37}$ C. P. Bean, Phys. Rev. Lett. 8, 250-253 (1962).

${ }^{38}$ U. Welp, W. K. Kwok, G. W. Crabtree, K. G. Vandervoort, and J. Z. Liu, Appl. Phys. Lett. 57, 84 (1990).

${ }^{39}$ T. Matsushita, J. Tanigawa, M. Kiuchi, A. Yamamoto, J.-i. Shimoyama, and K. Kishio, Jpn. J. Appl. Phys., Part 1 51, 123103 (2012).

${ }^{40}$ D. Dew-Hughes, Philos. Mag. 30, 293-305 (1974).

${ }^{41}$ A. M. Campbell, J. E. Evetts, and D. Dewhughes, Philos. Mag. 18, 313 (1968).

${ }^{42}$ R. Griessen, H.-h. Wen, A. J. J. van Dalen, B. Dam, J. Rector, H. G. Schnack, S. Libbrecht, E. Osquiguil, and Y. Bruynseraede, Phys. Rev. Lett. 72, 1910-1913 (1994).

${ }^{43}$ K. Kimura, M. Kiuchi, E. S. Otabe, T. Matsushita, S. Miyata, A. Ibi, Y. Yamada, and Y. Shiohara, Physica C 463-465, 697-701 (2007). 